\title{
openheart Comparative evaluation of coronary disease burden: bicuspid valve disease is not atheroprotective
}

\author{
Onur Baris Dolmaci (D) , ${ }^{1,2}$ Antoine H G Driessen, ${ }^{2}$ Robert J M Klautz, ${ }^{1,2}$ \\ Robert Poelmann, ${ }^{3}$ Jan H N Lindeman, ${ }^{4}$ Nimrat Grewal (1) ${ }^{1,5}$
}

To cite: Dolmaci OB, Driessen AHG, Klautz RJM, et al. Comparative evaluation of coronary disease burden: bicuspid valve disease is not atheroprotective. Open Heart 2021;8:e001772. doi:10.1136/ openhrt-2021-001772

JHNL and NG contributed equally.

Received 20 July 2021 Accepted 30 August 2021

Check for updates

(C) Author(s) (or their employer(s)) 2021. Re-use permitted under CC BY-NC. No commercial re-use. See rights and permissions. Published by BMJ.

${ }^{1}$ Cardiothoracic Surgery, Leiden University Medical Center, Leiden, The Netherlands ${ }^{2}$ Cardiothoracic Surgery, Amsterdam UMC Locatie AMC Amsterdam, The Netherlands ${ }^{3}$ Animal Sciences and Health, Leiden University Institute of Biology, Leiden, The Netherlands ${ }^{4}$ Vascular Surgery, Leiden University Medical Center, Leiden, The Netherlands ${ }^{5}$ Anatomy and Embryology, Leiden University Medical Center, Leiden, The Netherlands

Correspondence to Dr Nimrat Grewal; n.grewal@ lumc.nl

\section{ABSTRACT}

Objective Bicuspid aortic valve (BAV) has been associated with less atherosclerosis as compared with tricuspid aortic valve (TAV) patients. It, however, remains unclear whether this reflects the older age of TAV patients and/or accumulation of atherosclerotic risk factors or that the BAV phenotype is atheroprotective. Therefore, we compared the atherosclerotic disease burden of BAV and TAV patients, with that of the general (age-matched) population.

Methods The prevalence of coronary artery disease (CAD) and CAD risk factors in BAV and TAV patients who underwent aortic valve surgery were compared with the Dutch general practitioners registry data. BAV $(n=454)$ and TAV $(n=1101)$ patients were divided into four groups: BAV with aortic valve stenosis (BAVAoS), BAV with aortic valve regurgitation (BAV-AR), TAV with AoS (TAV-AoS) and TAV with AR (TAV-AR). The atherosclerotic disease burden of each group was compared with that of the corresponding age cohort for the general population.

Results CAD risk factors hypertension and hypercholesterolaemia were more prevalent in the surgery groups than the age-matched general population (all $p<0.001$ ). All BAVs (BAV-AoS and BAV$A R$ ) and TAV-AR had a similar incidence of CAD history as compared to the age-matched general populations $(p=0.689, p=0.325$ and $p=0.617$ respectively), whereas TAV-AoS had a higher incidence $(21.6 \%$ versus $14.9 \%$ in the age-matched general population, $p<0.001$ ). Conclusions Stenotic TAV disease is part of the atherosclerotic disease spectrum, while regurgitant TAV and all BAVs are not. Although the prevalence of cardiovascular risk factors is higher in all BAV patients, the prevalence of $C A D$ is similar to the general population.

\section{INTRODUCTION}

Bicuspid aortic valve (BAV) is the most common congenital cardiac anomaly, and associates with an increased risk for aortic valve abnormalities (ie, stenosis and regurgitation) and ascending aortic dilatation. ${ }^{1}$ The aetiology underlying thoracic aortic wall pathology in BAV has not been

\section{Key questions}

What is already known about this subject?

- Bicuspid aortic valve (BAV) is associated with significantly less atherosclerosis as compared with patients with a tricuspid aortic valve (TAV).

What does this study add?

- Atherosclerotic disease burden in BAV patients has until been studied in hospital populations with an increased cardiovascular disease burden. In this study, we, therefore, considered a comparison of the atherosclerotic disease burden of BAV and TAV patients, with that of the general (age-matched) population relevant.

How might this impact on clinical practice?

- Despite a higher prevalence of cardiovascular risk factors in BAV, the prevalence of coronary artery disease is similar to the general population. BAV disease is not a part of the atherosclerotic disease spectrum, whereas stenotic TAV disease is.

elucidated yet, most studies indicate a mechanism that is distinct from the aneurysm formation in tricuspid aortic valve (TAV) disease, which does not, or to a lesser extent involve aspects of the atherosclerotic process. ${ }^{2} 3$ Indeed, a comparison of the coronary artery atherosclerosis burden (coronary calcium scores) of BAV patients requiring aortic valve replacement with that of patients with a TAV showed a significantly lower coronary artery calcium burden in BAV patients. An observation which is consistent with less atherosclerosis in BAV patients. A critical question is whether these conclusions reflect the general older age of TAV patients and/or accumulation of atherosclerotic risk factors in TAV patients requiring aortic valve replacement/repair ${ }^{4}$ or, non-exclusively, that the BAV phenotype is athero-protective. ${ }^{35-8}$ To address these aspects, we considered a comparison of the atherosclerotic disease burden of BAV and 
TAV patients, with that of the general (age-matched) population relevant.

\section{METHODS}

\section{Study population}

The prevalence of coronary artery disease (CAD) and CAD risk factors in BAV and TAV patients were compared with the general population (Dutch general practitioners) registry data. ${ }^{9}$

Hospital patients were divided into four groups, namely: BAV with an aortic valve stenosis (BAV-AoS), BAV with an aortic valve regurgitation (BAV-AR), TAV with AoS (TAV-AoS) and TAV with AR (TAV-AR). Patients with a combined aortic valve vitium were included in either the stenotic or regurgitant group based on the most severe aortic valve pathology. All included hospital patients were surgically treated between 2006 and 2019 and were obtained from a retrospective study conducted at the Leiden University Medical Center (LUMC) in the Netherlands. ${ }^{3}$ Patient consent was waived. Subsequently, the atherosclerotic disease burden of each group was compared with that of the corresponding (matched) age cohort for the general population.

\section{Definitions}

Preoperative coronary angiographies were scored for all hospital patients, ${ }^{3}$ but could not be used to compare CAD with the general population since angiographies were not available for this group. Therefore, it was chosen to define $\mathrm{CAD}$ in the general population as a previous myocardial infarction or instable angina pectoris. Deduplication was performed for the hospital patients that had a history of both a myocardial infarction and instable angina pectoris. ${ }^{3}$

Cardiovascular risk factors included hypertension, diabetes mellitus (types 1 and 2) and hypercholesterolaemia. Current guidelines define hypertension as a blood pressure of $\geq 140 / 90 \mathrm{~mm} \mathrm{Hg}$. In the Netherlands Institute for Health Services Research (NIVEL) primary care database (NPCD) however hypertension is until defined as a blood pressure $\geq 160 / 95 \mathrm{~mm}$ Hg Therefore, hypertension was defined as a diastolic pressure of $\geq 95 \mathrm{~mm}$ $\mathrm{Hg}$, a systolic pressure of $\geq 160 \mathrm{~mm} \mathrm{Hg}$ on two separate occasions, or as the usage of antihypertensive drugs to be able to compare the study groups. ${ }^{9}$ Hypercholesterolaemia was scored if the patient had a total cholesterol level of $\geq 6.5 \mathrm{mmol} / \mathrm{L}$ or used lipid-lowering medications. ${ }^{9}$ Diabetes was defined as either a blood glucose level of $\geq 7.0 \mathrm{mmol} / \mathrm{L}$ on two separate (fasted) occasions, a glucose level of $\geq 11.1 \mathrm{mmol} / \mathrm{L}$ plus symptoms of hyperglycaemic or use of antidiabetic medication.

\section{Data sources}

Data from general practitioners participating in the NPCD, 2019 were used. The NPCD is a longitudinal database in which data from Dutch general practitioners is collected for study purposes. The database provides a representative sample of the Dutch population. ${ }^{9}$ Included diagnoses were coded by the primary care physicians using the International Classification of Primary Care. ${ }^{9}$

Table 1 Characteristics and prevalence of CAD and CAD risk factors of the hospital population $\mathrm{n}(\%)$

\begin{tabular}{|c|c|c|c|c|}
\hline & \multicolumn{4}{|c|}{ Aortic valve morphology } \\
\hline & \multicolumn{2}{|c|}{ Bicuspid aortic valves } & \multicolumn{2}{|c|}{ Tricuspid aortic valves } \\
\hline & Aos & AR & Aos & AR \\
\hline Characteristic & $n=365$ & $n=89$ & $n=931$ & $\mathrm{n}=170$ \\
\hline Male sex & $248(67.9)$ & $75(84.3)$ & $575(61.8)$ & $118(69.4)$ \\
\hline Age at surgery (years $\pm S D$ ) & $63 \pm 9.9$ & $53.8 \pm 10.6$ & $71.9 \pm 8.3$ & $64.7 \pm 11.4$ \\
\hline Body mass index $\left(\mathrm{kg} / \mathrm{m}^{2}\right)$ & $26.3(24-29.1)$ & $25.9 \pm 4.3$ & $26.8(24.5-30.1)$ & $26.2(23.2-28.4)$ \\
\hline Smoking status & $361 / 365^{\star}$ & $84 / 89^{*}$ & $884 / 931^{*}$ & $161 / 170^{*}$ \\
\hline Never & $161(44.1)$ & $44(49.4)$ & $422(45.3)$ & $79(46.5)$ \\
\hline Former & $119(32.6)$ & $16(18)$ & $331(35.6)$ & $44(25.9)$ \\
\hline Current & $81(22.2)$ & $24(27)$ & $131(14.1)$ & $38(22.4)$ \\
\hline Family history of CAD & $\begin{array}{l}346 / 365^{\star} \\
48(13.2)\end{array}$ & $\begin{array}{l}83 / 89^{*} \\
11(12.4)\end{array}$ & $\begin{array}{l}866 / 931^{*} \\
136(14.6)\end{array}$ & $\begin{array}{l}152 / 170^{*} \\
20(11.8)\end{array}$ \\
\hline Diabetes & $52(14.2)$ & $2(2.2)$ & $253(27.2)$ & $16(9.4)$ \\
\hline Hypertension & $179(49)$ & $51(57.3)$ & $619(66.5)$ & $113(66.5)$ \\
\hline Hypercholesterolaemia & $105(28.8)$ & $19(21.3)$ & $415(44.6)$ & $43(25.3)$ \\
\hline History of CAD & $32(8.8)$ & $2(2.2)$ & $201(21.6)$ & $18(10.6)$ \\
\hline
\end{tabular}

Hypertension is defined as a systolic blood pressure $>160 \mathrm{~mm} \mathrm{Hg}$. Hypercholesterolaemia: total cholesterol level of $\geq 6.5 \mathrm{mmol} / \mathrm{L}$ or use of lipid-lowering medication. Diabetes: as a blood glucose level of $\geq 7.0 \mathrm{mmol} / \mathrm{L}$ on two separate (fasted) occasions, a glucose level of $\geq 11.1$ $\mathrm{mmol} / \mathrm{L}$ plus symptoms of hyperglycaemia or usage of antidiabetic medication.

${ }^{*}$ Denominator represents variables with incomplete data.

AoS, aortic valve stenosis; AR, aortic regurgitation; BAV, bicuspid aortic valve; ; CAD, coronary artery disease. 
Data regarding the BAV and TAV hospital patients were from a single centre cohort of 1555 (454 BAV, $1101 \mathrm{TAV}$ ) patients who underwent aortic valve repair or replacement. ${ }^{3}$ The medical history of these patients were all re-evaluated for the prevalence of $\mathrm{CAD}$ and risk factors.

\section{Patient and public involvement}

Patients were not involved

\section{Statistical analysis}

Continuous data are presented as mean and SD, categorical data as frequencies and percentages. The Fischer's exact test was used for analysing categorical data. All variables with a $p<0.2$ in the univariate analyses were included in a multivariate model in order to correct the differences between the groups (eg, age, sex and date of surgery). A p $<0.05$ was considered to be significant. All statistical analyses were conducted using IBM SPSS for Windows V.25.0.

\section{RESULTS}

\section{Cardiovascular burden: BAV versus TAV patients}

Baseline and CAD-related factors of the hospital population are summarised in table 1.

Cardiovascular risk factors were more common in TAV patients (AoS and AR) than in BAV patients (AoS and AR) (all $\mathrm{p}<0.001$ ). Similarly, TAV patients were more likely to have a history of CAD (OR: 3.07 (95\% CI 2.10 to 4.48); $\mathrm{p}<0.001)$. This difference remained after correction for age, sex and date of surgery (corrected $\mathrm{OR}_{(}$
: 2.73 (95\% CI 1.83 to 4.09$) ; \mathrm{p}<0.001)$. Comparison of echocardiographic characteristics were already made in a previous study, which showed higher aortic valve gradients in BAV patients (peak gradient of 73 vs $62 \mathrm{~mm} \mathrm{Hg}$, $\mathrm{p}<0.001){ }^{3}$

Subanalysis showed a higher prevalence of risk factors hypercholesterolaemia and diabetes mellitus in TAVAoS vs TAV-AR (both $\mathrm{p}<0.001$ ), as well as with a higher prevalence of previous $\mathrm{CAD}$ (corrected $\mathrm{OR}_{\text {(age and sex) }}$ for TAV-AR vs TAV-AoS: 1.08 (95\% CI 1.06 to 1.10 ); $\mathrm{p}=0.001$ ). Comparison of BAV-AoS patients with BAV-AR patients only showed a higher prevalence of diabetes mellitus in BAV-AoS patients $(\mathrm{p}<0.001)$.

\section{Cardiovascular burden: BAV and TAV patients versus general population}

The prevalence of cardiovascular risk factors and CAD in the patients and the respective reference (age-matched) general populations are summarised in figure 1 .

With the exception of hypercholesterolaemia in the TAV-AR group, hypertension and hypercholesterolaemia were more prevalent in the surgery groups than in the age-matched general population (all $\mathrm{p}<0.001$ ).

Compared with the general population, a diagnosis of diabetes mellitus was more common in the TAVAoS group $(p \leq 0.001)$, less common in the TAV-AR and BAV-AR groups (9\% vs $16.9 \%, \mathrm{p}<0.001$ resp. $2.2 \%$ vs $6.6 \%, \mathrm{p} \leq 0.001)$ and similar in the BAV-AoS group.

The prevalence of a history of CAD was similar in BAV patients (both BAV-AoS and BAV-AR), TAV-AR patients,
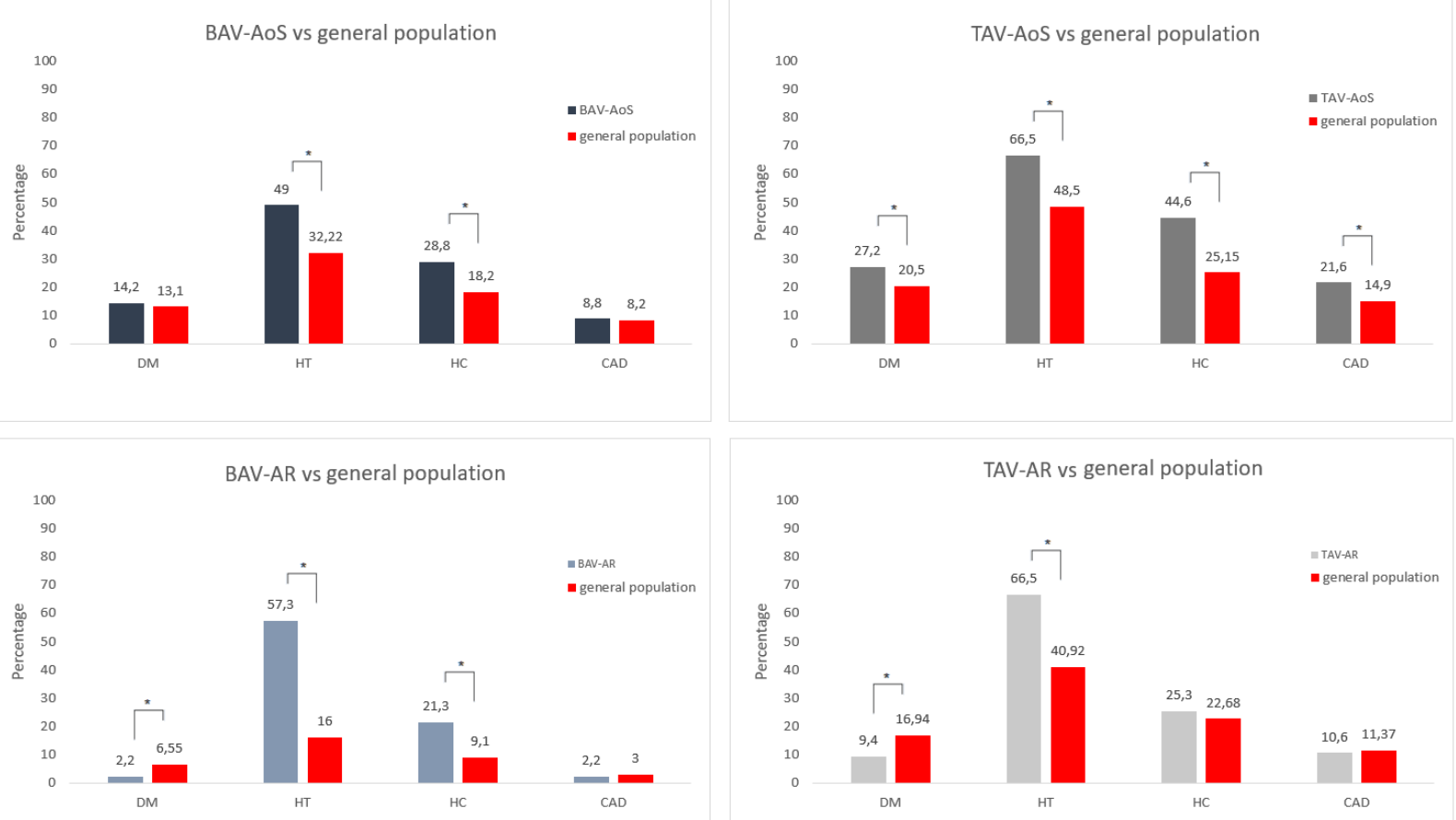

Figure 1 Comparison of $C A D$ and $C A D$ risk factors in BAV and TAV groups versus the age-matched and sex-matched general population. AR, aortic regurgitation; AoS, aortic valve stenosis; BAV, bicuspid aortic valve; CAD, coronary artery disease; DM, diabetes mellitus; $\mathrm{HC}$, hypercholesterolaemia; $\mathrm{HT}$, hypertension; TAV, tricuspid aortic valve; ${ }^{\star} \mathrm{P} \leq 0.001$. 
and the age-matched general populations $(\mathrm{p}=0.689$, $\mathrm{p}=0.325$ and $\mathrm{p}=0.617$, respectively). In contrast, TAV-AoS associated with a higher incidence of a history of CAD (21.6\% vs $14.9 \%$ in the age-matched general population, $\mathrm{p}<0.001)$.

\section{DISCUSSION}

The findings of this study characterise stenotic TAV disease as a part of the atherosclerotic disease spectrum (based on cardiovascular risk factors and more prevalent CAD compared with the general population), while TAV-AR and BAV (both AR and AoS) do not. Although the prevalence of cardiovascular risk factors was higher in the BAV groups, the prevalence of CAD was similar to the general population.

The relationship of CAD and the aortic valve morphology and a possible atheroprotective effect of BAV is a topic of ongoing debate..$^{5-8}$ Previous studies have shown a significantly larger aortic valve calcium volume in BAV patients than TAV patients, ${ }^{5}$ but strikingly a lower prevalence of cardiovascular risk factors, coronary calcification, CAD and coronary revascularisation. ${ }^{36-8}$ These observations imply that the aortic valve calcifications in BAV disease merely reflect local degenerative changes rather than systemic atherosclerotic changes. ${ }^{36}$

Our previous report focusing on an in-hospital population implied a protective relationship between BAV and atherosclerosis. ${ }^{3}$ The similar prevalence of CAD in BAV patients and the general population, and the increased prevalence of CAD in TAV-AoS patients observed in this study suggests that the conclusions with regard to a possible atheroprotective phenotype of BAV are interfered by the strong association of atherosclerosis and TAV-AoS disease. Nevertheless, BAV patients present with a more unfavourable cardiovascular risk profile than the general population. This observation could support an atheroprotective phenotype in BAV disease, yet it cannot be excluded that the higher prevalence of risk factors reflects a referral phenomenon with increased awareness on the assessment and treatment of cardiovascular risk factors in the clinical setting.

\section{LIMITATIONS}

Due to incomplete data of the general population not all cardiovascular risk factors such as laboratory findings, tobacco usage or family history of CAD could be included in this study. Available data of the general population might be biased by aspects such as under-reporting, a more systematic screening for risk factor in the hospital population and a reliance on coding (NPCD). Nevertheless, it is important to point out that the coding of general practitioners have improved significantly in the last few years. ${ }^{9}$ Comparisons of CAD were made based solely on chart review, as coronary angiographies were not available for the general population. Finally, the definition of hypertension $(>160 \mathrm{~mm} \mathrm{Hg})$ used in this study was dictated by NPCD. This definition is higher than the consensus threshold, and may consequently lead to under-reporting of hypertension in the general population. We consider it unlikely that this higher threshold influences the conclusions with regard to the hospital population, since these diagnoses were all based on hypertensive use and not on actual blood pressure.

\section{CONCLUSION}

BAV patients and TAV-AR present with a worse cardiovascular risk profile than, but similar prevalence of CAD as the general population. These conclusions do not characterise BAV as atheroprotective. On the other hand, the higher prevalence of cardiovascular risk factors and $\mathrm{CAD}$ in TAV-AoS patients characterises TAV-AoS as a part of the atherosclerotic spectrum of diseases.

Contributors JHNL, NG and OBD conceived of and planned the paper, carried out analysis of the results and wrote the manuscript; All authors reviewed and edited the manuscript. JHNL and NG are responsible for the overall content as guarantors.

Funding The authors have not declared a specific grant for this research from any funding agency in the public, commercial or not-for-profit sectors.

Competing interests None declared.

Patient consent for publication Not required.

Ethics approval Approved by the medical ethics committee of the Leiden University Medical Center (METC LDD, case number G19.113).

Provenance and peer review Not commissioned; internally peer reviewed. Data availability statement Data are available on reasonable request.

Open access This is an open access article distributed in accordance with the Creative Commons Attribution Non Commercial (CC BY-NC 4.0) license, which permits others to distribute, remix, adapt, build upon this work non-commercially, and license their derivative works on different terms, provided the original work is properly cited, appropriate credit is given, any changes made indicated, and the use is non-commercial. See: http://creativecommons.org/licenses/by-nc/4.0/.

ORCID iDs

Onur Baris Dolmaci http://orcid.org/0000-0002-0578-6407

Nimrat Grewal http://orcid.org/0000-0001-9981-8336

\section{REFERENCES}

1 Ward C. Clinical significance of the bicuspid aortic valve. Heart 2000;83:81-5.

2 Mordi I, Tzemos N. Bicuspid aortic valve disease: a comprehensive review. Cardiol Res Pract 2012;2012:1-7.

3 Dolmaci OB, Legué J, Lindeman JHN, et al. Extent of coronary artery disease in patients with stenotic bicuspid versus tricuspid aortic valves. J Am Heart Assoc 2021;10:e020080.

4 Weisenberg D, Sahar Y, Sahar G, et al. Atherosclerosis of the aorta is common in patients with severe aortic stenosis: an intraoperative transesophageal echocardiographic study. J Thorac Cardiovasc Surg 2005;130:29-32.

5 van Rosendael PJ, Kamperidis V, Kong WKF, et al. Comparison of quantity of calcific deposits by multidetector computed tomography in the aortic valve and coronary arteries. Am J Cardiol 2016;118:1533-8.

6 Jackson V, Eriksson MJ, Caidahl K, et al. Ascending aortic dilatation is rarely associated with coronary artery disease regardless of aortic valve morphology. J Thorac Cardiovasc Surg 2014;148:2973-80.

7 Agnese V, Pasta S, Michelena HI, et al. Patterns of ascending aortic dilatation and predictors of surgical replacement of the aorta: a comparison of bicuspid and tricuspid aortic valve patients over eight years of follow-up. J Mol Cell Cardiol 2019;135:31-9.

8 Cozijnsen L, van der Zaag-Loonen HJ, Cozijnsen MA, et al. Differences at surgery between patients with bicuspid and tricuspid aortic valves. Neth Heart J 2019;27:93-9.

9 NIVEL zorgregistraties eerste lijn (Coronaire hartziekten 2019 B, diabetes mellitus 2019 and cholesterol, 2020. Available: https://www. volksgezondheidenzorg.info [Accessed 23 Mar 2021]. 